\title{
Interferometric and nonlinear-optical spectral-imaging techniques for outer space and live cells
}

Kazuyoshi Itoh

Kazuyoshi Itoh, "Interferometric and nonlinear-optical spectral-imaging techniques for outer space and live cells," Proc. SPIE 9792, Biophotonics Japan 2015, 979203 (9 December 2015); doi: 10.1117/12.2214994

SPIE. Event: SPIE/OSJ Biophotonics Japan, 2015, Tokyo, Japan 


\title{
Interferometric and nonlinear-optical spectral-imaging techniques for outer space and live cells
}

\author{
Kazuyoshi Itoh \\ Science Technology Entrepreneurship Laboratory (E-Square), Osaka University \\ 2-1 Yamada-oka, Suita, Osaka, 565-0871 Japan
}

\begin{abstract}
Multidimensional signals such as the spectral images allow us to have deeper insights into the natures of objects. In this paper the spectral imaging techniques that are based on optical interferometry and nonlinear optics are presented. The interferometric imaging technique is based on the unified theory of Van Cittert-Zernike and Wiener-Khintchine theorems and allows us to retrieve a spectral image of an object in the far zone from the 3D spatial coherence function. The retrieval principle is explained using a very simple object. The promising applications to space interferometers for astronomy that are currently in progress will also be briefly touched on. An interesting extension of interferometric spectral imaging is a 3D and spectral imaging technique that records 4D information of objects where the 3D and spectral information is retrieved from the cross-spectral density function of optical field. The 3D imaging is realized via the numerical inverse propagation of the cross-spectral density. A few techniques suggested recently are introduced. The nonlinear optical technique that utilizes stimulated Raman scattering (SRS) for spectral imaging of biomedical targets is presented lastly. The strong signals of SRS permit us to get vibrational information of molecules in the live cell or tissue in real time. The vibrational information of unstained or unlabeled molecules is crucial especially for medical applications. The 3D information due to the optical nonlinearity is also the attractive feature of SRS spectral microscopy.
\end{abstract}

Keywords: Interferometric imaging, Michelson's stellar interferometer, Radio interferometry, spectral-imaging, 3D imaging, nonlinear optics, stimulated Raman scattering, biomedical optics

\section{INTRODUCTION}

Albert A. Michelson succeeded in determining the diameter of Betelgeuse ( $\alpha$ Orionis) by using the Mount Wilson Observatory's 100-inch telescope with the help of the movable outer mirrors in 1921 [1]. The interference fringe formed in the focal plane of the telescope moved by air turbulence. But the moving fringe was visible and its contrast was perceptible. From the separation of the outer mirrors where the fringe contrast disappeared he determined the diameter of the fixed star. It is important to note that the images were not reconstructed due to the air turbulence. Here I will explain the principle of image reconstruction by interferometers and the problems in optical interferometers in the following and then its extensions to 3D and spectral imaging [2]. I also introduce you the recent result of the nonlinear-optical spectralimaging technique of the stimulated Raman scattering (SRS) microscopy [3-5].

\subsection{The success of radio interferometers and problems in optical interferometric spectral image}

The principle of interferometeric image reconstruction is based on the Van Cittert-Zernike (VCZ) Theorem [6]. If you measure the spatial coherence function over the lateral plane with regard to the line of sight, the image intensity distribution is given by its 2D Fourier transform. Since the coherence function is an analytic function and the measurement of its imaginary part or the phase angle is crucial for the direct image reconstruction. Unfortunately, the air turbulence disturbs the phase of optical coherence function as is implied by the motion of optical fringe disturbed by the air turbulence. On the other hand, the radio interferometers are relatively free from the air turbulence and images are readily reconstructed. In the radio interferometers the radio signals are directrly detected by the antennas and the electric signals can be cabled to the desired locations and the coherence functions can be obtained by using the electronic correlators [7]. High-resolution multispectral imaging can also be carried out by the radio interferometers, because if they are equipped with the electronic time-delay circuit, they can output the coherence function with the time delay from which we can retrieve the cross-spectral density function by the Fourier transform relationship. Since the cross-spectral

Biophotonics Japan 2015, edited by Takashige Omatsu, Yoshio Hayasaki, Yusuke Ogura, Yasuyuki Ozeki, Seigo Ohno, Proc. of SPIE Vol. 9792, 979203 - (c) 2015 SPIE · CCC code: 1605-7422/15/\$18 · doi: 10.1117/12.2214994 
density function is the spectral component of the coherence function, the high-resolution multispectral imaging is straightforward. The temporal correlation function or the coherence function for each base line is usually measured first by an analogue or digital correlator depending on the generations and the cross-power or cross-correlation functions is calculated by the Fourier transform relationship. Y. Chikada et al. constructed a modern FFT spectrum analyzer with the highly parallel pipeline architecture where the digitized radio signals are numerically Fourier transformed before the correlation process [8]. The practical problems in optical interferometry are the weakness in air turbulence and the difficulty in high-resolution multi-spectral imaging.

\section{THE FOURIER-TRANSFORM SPECTRAL IMAGING TECHNIQUE}

If you properly combine the Michelson stellar interferometer and Michelson-Morley interferometer, you can obtain the coherence function with the long time delay and reconstruct a high-resolution spatio-spectral image, which is a twodimensional (2D) array of high-resolution spectra at each location over the object surface. The principle is based on a Fourier-transform relationship derived from the basic propagation formula describing the field correlation function in terms of the source correlation function [9] and can be considered as a unification of the VCZ and Wiener-Khinchine theorems. The theory was published in 1986 with a simple experiment [10]. Shortly after, Mariotti and Ridgway suggested a simple and quite effective combination of these interferometers for use in astronomy [11]. The Longbaseline ground-based type of this spatio-spectral interferometers using optical single-mode fibers $[12,13]$ and a similar type of ground-based system without the use of single-mode fibers $[14,15]$ are under study. In the following, the author explains the principle of the Fourier-transform spectral imaging technique simply if at all possible and then introduce briefly the current status of the Fourier-transform spectral imaging techniques in space for astronomy [16-20].

\subsection{Principle}

The spectral imaging technique to be discussed here is, as already mentioned, based on the unified theory combining the principles of the Michelson's two well-known interferometers. The unified theory states that if you can measure the 3D spatial coherence function of optical disturbances coming from the far zone you can retrieve at one sweep both of the spatial and spectral information of the light source. The principle will be explained in an intuitive and comprehensible way using a simple light source model in the following. Let us consider the 3D spatial coherence of optical field illuminated by a simple object in the far zone. Here we assume two point sources as shown in Fig. 1. The two point sources in the $z=0$ plane emit mutually incoherent quasi-monochromatic lights. Here, "incoherent" means the lights from the two point sources are statistically independent and "quasi-monochromatic" means that the spectral width of the light is very small compared with the average frequency. For simplicity we assume that the observation area is located sufficiently far from the light sources and the optical waves coming from the two light sources can be considered to have flat or planar wavefronts. We also assume to focus on a certain component of polarization and deal with the optical field as scalar.

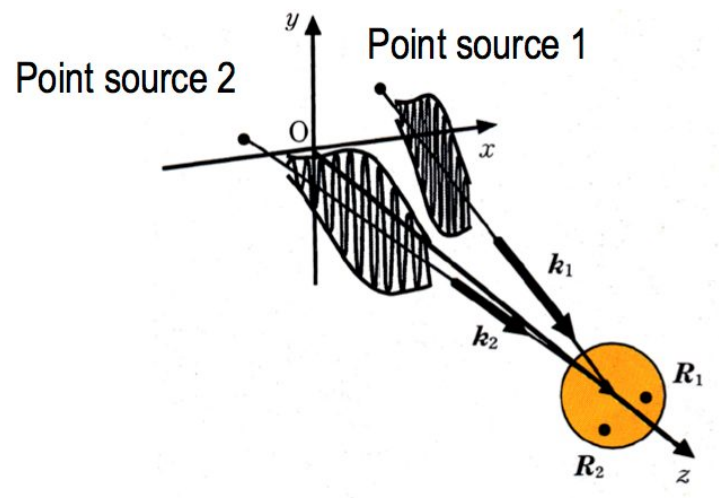

Fig. 1. A simple model; Optical field is generated by the two point sources in the far zone. 
Let the two point sources be numbered $(j=1,2)$ and the mean 3D wavenumbers and mean angular frequencies be $\overline{\boldsymbol{k}}_{j}$ and $\bar{\omega}_{j}=c \bar{k}_{j}$, respectively. Then, the optical wave field at the location $\boldsymbol{R}$ in the observation area at time $t$ is given by the summation of two plane waves with a slowly-varying envelope functions that changes very slowly compared with the mean frequency or mean wavelength $V_{j}\left(\overline{\boldsymbol{k}}_{j} \cdot \boldsymbol{R}-\bar{\omega}_{j} t\right),(j=1,2)$. Then the coherence function is given by [2]

$$
J\left(\boldsymbol{R}_{1}, \boldsymbol{R}_{2}\right) \equiv \Gamma\left(\boldsymbol{R}_{1}, \boldsymbol{R}_{2} ; 0\right)=\left\langle\left|V_{1}\right|^{2}\right\rangle \exp \left[i \overline{\boldsymbol{k}}_{1} \cdot\left(\boldsymbol{R}_{2}-\boldsymbol{R}_{1}\right)\right]+\left\langle\left|V_{2}\right|^{2}\right\rangle \exp \left[i \overline{\boldsymbol{k}}_{2} \cdot\left(\boldsymbol{R}_{2}-\boldsymbol{R}_{1}\right)\right]
$$

where * denotes the complex conjugate, $\langle\ldots\rangle$ means the statistical ensemble average, and the temporal stationarity of the optical field is assumed and the ensemble average can usually be considered to be replaceable with the time average.

From Eq. (1), we understand that the two mutually independent point sources produces in the far field the coherence function that depends only on the relative positions $\left(\boldsymbol{R}_{2}-\boldsymbol{R}_{1}\right)$ and produces a pair of uniform complex fringes. This is the typical coherence function produced by a couple of fixed stars in the night sky in front of our eyes. The period and direction of the complex fringe are determined by each 3D wave vector, $\boldsymbol{k}_{j}(\mathrm{j}=1$ or 2$)$, the amplitude of the fringe being proportional to the brightness of the point source. It is now clear that if we Fourier transform Eq. (1), we get in the wave vector space the delta-like functions located at $\overline{\boldsymbol{k}}_{j}$ with the strength proportional to $\left\langle\left|V_{j}\right|^{2}\right\rangle$. Every luminous objects or objects illuminated by substantially incoherent light can be considered as a collection of mutually independent light sources, the above discussion directly applies to general extended incoherent objects.

The relationship between the incoherent light source and the coherence function in the far zone has been explained by using the simple light source model. But for general discussions, the propagation of cross-spectral density is adequate. Itoh and Ohtsuka [10] derived a general formula resting on the work by Carter and Wolf [9] as

$$
J\left(\boldsymbol{R}_{1}, \boldsymbol{R}_{2}\right) \equiv \int d \omega d^{2} r_{\perp} S_{\omega}\left(\boldsymbol{r}_{s \perp}, 0\right) \frac{\exp \left[i \overline{\boldsymbol{k}} \cdot\left(\left|\boldsymbol{R}_{2}-\boldsymbol{r}_{\perp}\right|-\left|\boldsymbol{R}_{1}-\boldsymbol{r}_{\perp}\right|\right)\right]}{\left|\boldsymbol{R}_{1}-\boldsymbol{r}_{\perp} \| \boldsymbol{R}_{2}-\boldsymbol{r}_{\perp}\right|},
$$

where $S_{\omega}\left(\boldsymbol{r}_{s \perp}, 0\right)$ is the spectral intensity distribution of the incoherent light source in the 2D object plane at a point $\boldsymbol{r}_{s \perp}$. Equation (4) includes both of the spatial and spectral information of object and can be considered as the extension of the VCZ theorem to polychromatic objects.

They derived under appropriate conditions the following equation:

$$
J(\boldsymbol{\rho})=\int d^{3} k G(\boldsymbol{k}) \exp (i \boldsymbol{k} \cdot \boldsymbol{\rho})
$$

where

$$
J(\boldsymbol{\rho})=J(\boldsymbol{R}, \boldsymbol{R}+\boldsymbol{\rho}), \quad G(\boldsymbol{k})=c\left(k k_{z}\right)^{-1} S_{\omega}\left(\boldsymbol{r}_{s \perp}(\boldsymbol{k}), 0\right) .
$$

They showed that from the Fourier inversion relationship the function $G(\boldsymbol{k})$ can be retrieved from the coherence function $J(\rho)$ in the observation area, and named the function $G(\boldsymbol{k})$, the spectral image. Eq. (3) is said to unify the VCZ and Wiener-Khinchine theorems.

Figure 2 shows the 2D spectral image reconstructed experimentally [10, 16]. A point object with two spectral components was produced by focusing a light beam composed of $\mathrm{He}-\mathrm{Cd}$ (4416-A) and He-Ne (6328-A) laser spectra. You can see the two spectral components lie on the same radial line, which indicates the angular position of object. The authors also discussed briefly on the signal to the noise ratio for astronomical observation of stars. 


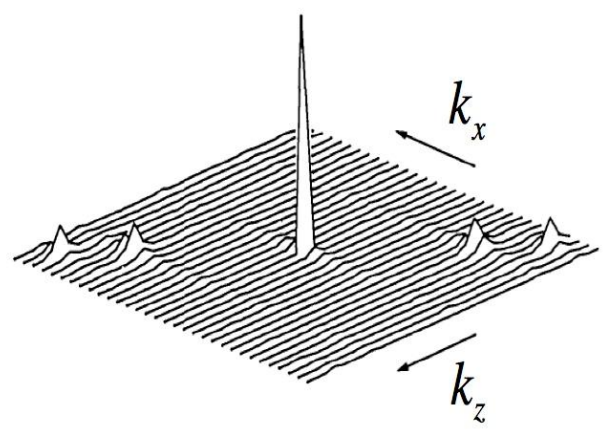

Fig. 2. Reconstructed spectral image of a point object composed of He-Cd (4416-A) and He-Ne (6328-A) laser spectra.

\subsection{Double-Fourier spatio-spectral interfereometry}

The double-Fourier spatio-spectral technique was suggested in 1988 assuming aperture synthesis for the very high spatial and spectral resolution [11]. The concept of the double Fourier interferometer is shown in Fig. $3[11,16]$. As can be seen, the interferometer is a simple and effective combination of the two famous interferometers due to Michelson. The 3D spatial shear is introduced into the incoming optical field and spectral image is retrieved by the principle of the Fouriertransform spectral imaging.

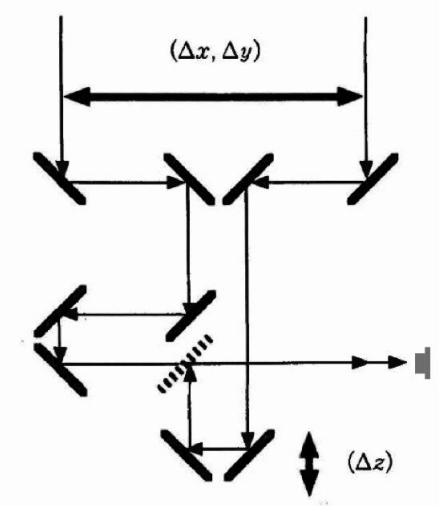

Fig. 3. The concept of the double Fourier interferometer.

Note that the path-difference introduced into the direction of the optical axis truly introduced to create 3D shear into the field not to create the time shear into the field as is expected in the simple Fourier spectrometry. This is the district difference between the Fourier-transform spectral imaging and the spatio-spectral radio interferometry or long-baseline spatio-spectral optical interferometry using the single-mode optical fibers. The latter ones generate the cross-spectral density by introducing the time delay into the incoming optical fields at two positions with the diffraction limited spatial resolution of the two telescopes or the siderostats.

\subsection{Resolution limit and field of view}

The resolution limit of the Fourier transform spectral imaging technique is briefly described here [10]. Let the measurement range of $\rho$ of $J(\rho)$ be $\Delta \rho=\left(L_{x}, L_{y}, L_{z}\right)$. Then, the spatial lateral resolution about $k$ is given by $\delta k_{x}=1 / L_{x}, \delta k_{y}=1 / L_{y}$ and the spectral resolution is approximately given by $\delta k_{z}=1 / L_{z}$. On the other hand, the field of view of is limited by the size of detection cell for $J(\rho)$. If the detection cell size is given by $\delta \rho=\left(l_{x}, l_{y}, l_{z}\right)$, 
the field of view is given by $\Delta k_{x}=1 / l_{x}, \Delta k_{y}=1 / l_{y}$ and the detectable spectral range is spproximately given by $\Delta k_{z}=1 / l_{z}$. The resolution limit and the field of view of the double Fourier technique are determined in the same way.

\subsection{Projects for high-resolution spectral imaging in space - WIIT, BETTII, SPIRIT, and SPECS -}

Several projects of space-borne optical or infrared interferometers are also in progress [17-19]. The SPIRIT [17] will have the high angular resolution $(0.3 \mathrm{arcsec})$ and the detection wavelength ranges from 25 to $400 \mu \mathrm{m}$. For details readers are referred to Ref. [17]. The authors believe that the space-borne optical interferometers are the most promising application of the Fourier-transform spectral imaging or double Fourier technique.

The SPIRIT is a space-based spatio-spectral interferometer based on the Fourier-transform spectral imaging or the double-Fourier technique [17]. The aperture synthesis technique [11] provides us much more spatial resolution than the single disc telescope. Figure 4 shows the concept of the Space Infrared Interferometric Telescope (SPIRIT). The SPIRIT is to provide sub-arcsecond resolution images and spectra with resolution $\mathrm{R}=3000$ in a 1 arcmin field of view in the wavelength range of $25-400 \mu \mathrm{m}$. The three primary scientific objectives of SPIRIT is as follows: to (1) learn how planetary systems form from protostellar disks, and how they acquire their inhomogeneous composition; (2) characterize the family of extrasolar planetary systems by imaging the structure in debris disks to understand how and where planets of different types form; and (3) learn how high-redshift galaxies formed and merged to form the present-day population of galaxies. The SPIRIT is to use the wide-field imaging technique employed in WIIT where a detector array was substituted for the usual single detector in the focal plane. Each detector element sees each area in the universe at the given 3D baseline and detects the 3D spatial coherence of incoming light in the parallel fashion [18]. Then, you can get multiple sets of fringe data for wider field of view simultaneously. This simple but very effective technique is to be used in the future interferometers such as SPIRIT and the Balloon Experimental Twin Telescope for Infrared Interferometry (BETTII) [20]. Please note that the SPIRIT is still a pathfinder to the Sub-millimeter Probe of the Evolution of Cosmic Structure (SPECS) [21] and the SPECS is conceived as a 1-kilometer maximum baseline far-IR interferometer requiring multiple spacecrafts flying in a tethered formation.

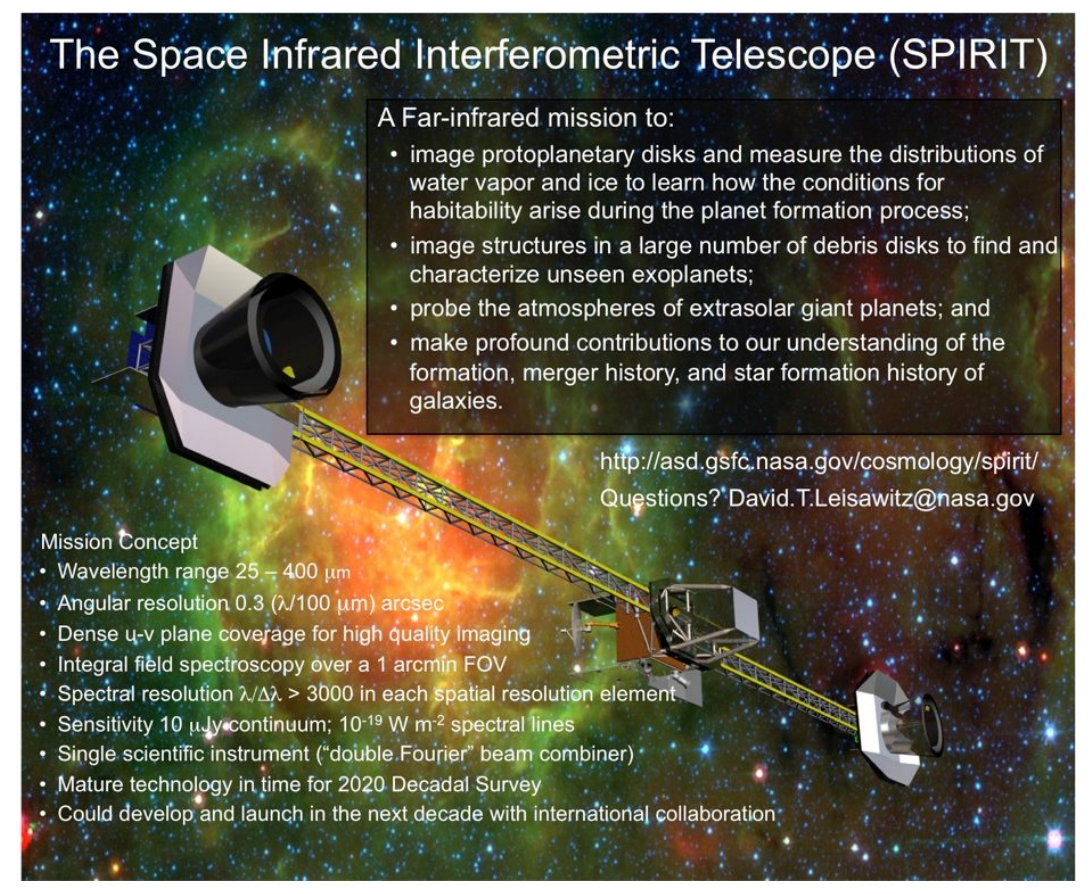

Fig. 4. The concept of the Space Infrared Interferometric Telescope (SPIRIT) (Courtesy of David Leisawitz of NASA Goddard Space Flight Center). 


\section{COHERENCE-BASED 3D AND SPECTRAL IMAGING TECHNIQUES}

Interferometric methods to obtain both the 3D spatial and the spectral information of a usual polychromatic incoherent object are forming the new modality of imagers. These techniques can be considered to be a new extension of the Fourier transform spectral imaging technique. Those suggested recently are briefly introduced [22-24].

\subsection{Inverse or Back Propagation of Cross-spectral Density for 3D imaging}

The idea of back-propagation of cross-spectral density was first suggested by Arimoto, Yoshimori and Itoh in 1999 [22]. The principle of the back-propagation of cross-spectral density is based on the formula derived in [6]. They employed the computationally less intensive propagation formula in the Fourier domain. They succeeded in reconstructing a 1D light source simulated by the He-Ne laser light and rotating diffuser behind a slit. They measured the real and imaginary parts of the 1D coherence function or the cross-spectral density using the wave-front type of interferometer and then back-propagated the cross-spectrum into the source plane. The cross-spectra in the intermediate planes were also visualized.

\subsection{Digital Holographic 3D Imaging Spectrometry}

Teeranutraont and Yoshimori suggested the digital holographic technique of 3D and spectral imaging and demonstrated the reconstruct experiment [23]. They first measured the asymmetric 3D coherence function of the optical field generated by the polychromatic objects illuminated by a natural light and they obtained the cross-spectral density by the Fourier transform relationship. They calculated the cross-spectral densities at the intermediate and the source planes by the backpropagation technique.

\subsection{Mach-Zehnder Radial-shearing Interferometer for 3D \& Spectral imaging}

Just recently, Naik, Pendrini, Takada and Osten demonstrated the Radial-Shearing technique for the fast 3D and spectral imaging [24]. Similarly to the previous work, they obtained first the shift-invariant cross-spectral density from the radially-sheared 3D coherence function via the Fourier transform relationship and then back-propagated the crossspectral density function with the radial shear. They used the Mach-Zehnder configuration and created the radial shear by using reversed order of lenses. The shear along the optical axis was introduced by the shift of the piezo-electric mirror. The phase errors were monitored by the He-Ne laser source and images of the red and green LEDs were clearly reconstructed. The advantage of their system is the efficiency of coherence detection.

\section{STIMULATED RAMAN SPECTRAL MICROSCOPY}

Recently, stimulated Raman scattering (SRS) microscopy has been reported as a technique for overcoming the nonresonant background limitation of CARS microscopy [3-5]. In SRS microscopy, we use two-color pulses, which are called pump and Stokes, respectively. One of the pulses is modulated temporally and both of the pulses are precisely overlapped in time, and collinearly and tightly focused on a sample. When the difference in frequency corresponds to the intrinsic molecular vibrational frequency, the optical energy of the pump pulse is transferred to the Stokes pulse as a result of coherent excitation of molecular vibration through SRS process. The resultant intensity modulation is transferred to the other pulse, which is detected by a lock-in amplifier. SRS offers several advantages such as quantitative contrast and accessibility to vibrational spectrum. Video rate imaging is achievable because the sensitivity of SRS microscopy at the shot noise limit is comparable to that of CARS microscopy [4].

\subsection{Principle of SRS microscopy}

The SRS microscopy offers novel capabilities of the label-free and high-speed imaging with the chemical contrast. Fast imaging is especially important for biomedical applications where the degradation due to the sample movement is crucial. In SRS microscopy, two color laser pulses at pump frequency $\omega_{\mathrm{AS}}$ and $\omega_{\mathrm{S}}$ are used, and either the pump or the Stokes beam is intensity modulated and both of the pulses are focused into the sample. Then, the SRS-induced intensity- 
modulation of the other beam is detected by a lock-in amplifier. Figure 5 shows the resultant field due to the SRS process. The higher frequency component of the side bands is the CARS (Coherent Anti-Stokes Raman Scattering) signal.

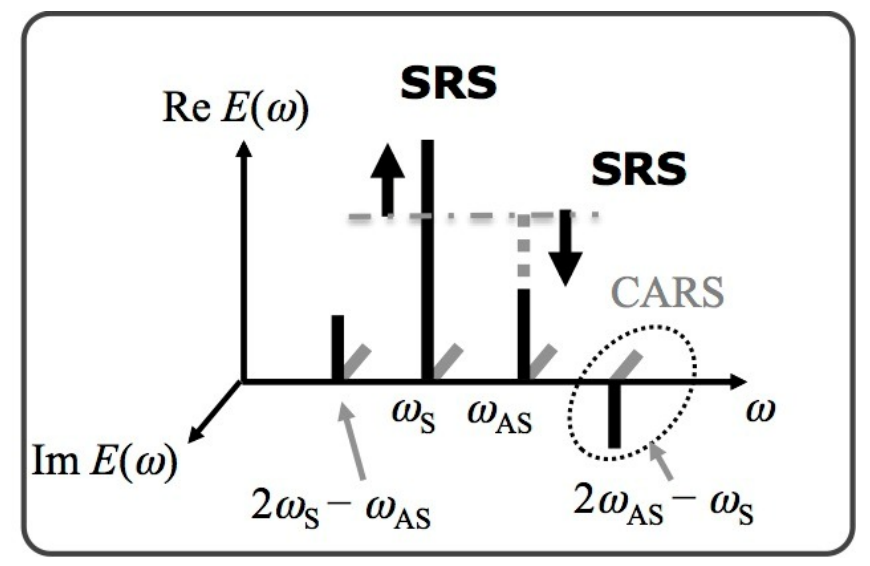

Fig. 5 Resultant field of the Stimulated Raman process.

\subsection{Experimental results}

Figure 2 shows the experimental setup for the fast SRS microsopy. A Ti:sapphire oscillator generated $76 \mathrm{MHz}, 797 \mathrm{~nm}$ pulses with a $4.8 \mathrm{ps}$ duration and a $\mathrm{Yb}$ fiber oscillator $(\mathrm{YbF})$ [26] generated $38 \mathrm{MHz}$ trains of broadband pulses at a central wavelength of $1030 \mathrm{~nm}$, a spectral width of $\sim 30 \mathrm{~nm}$. The YbF pulses were lead to TBPF (tunable band-pass filter), which consisted of a Galvanic Mirror, relay lenses, and a diffraction grating. The transmission bandwidth of the TBPF was as narrow as $\sim 0.3 \mathrm{~nm}$. The corresponding Gaussian pulses had a duration of $5.2 \mathrm{ps}$ at the Fourier-transform limit. Then, the filtered pulses were amplified by concatenated Yb-doped fiber amplifiers (YDFAs) to an average power of $\sim 300 \mathrm{~mW}$.

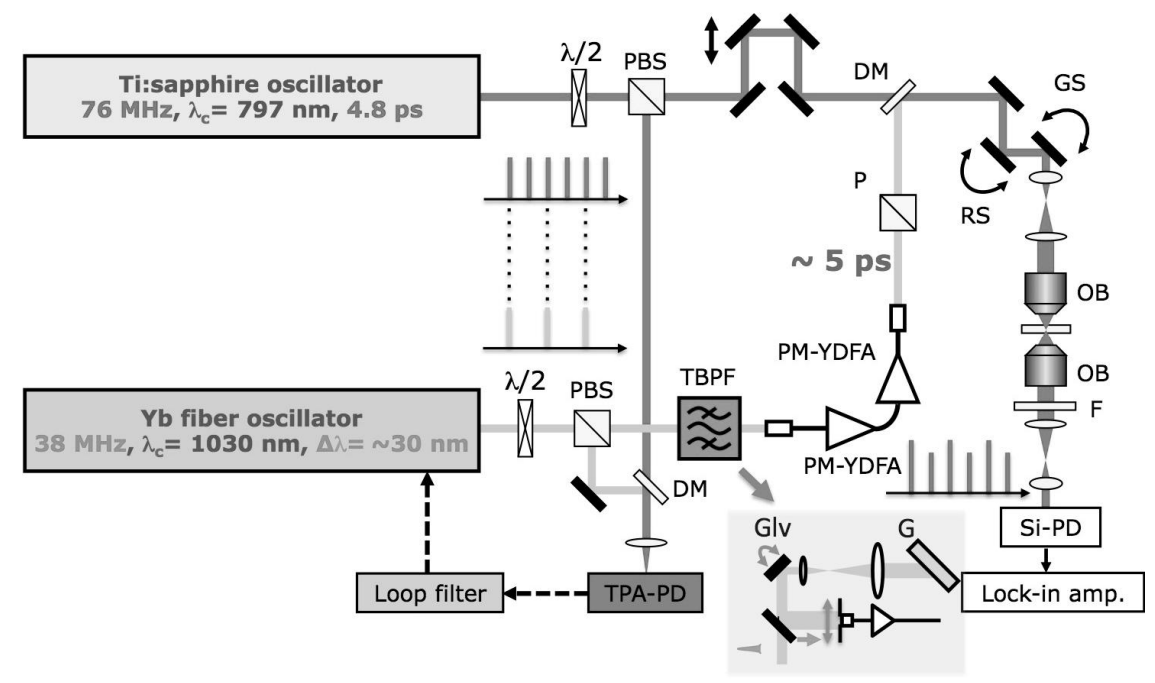

Fig. 6 Optical layout of SRS microscope system. 


\subsection{Experimental results}

A typical of the experimental results [27] is presented in Fig. 7. This is the colored composite image of a mouse pancreas tissue converted into a monochrome image. The three major components obtained by the ICA (Independent Component Analysis) technique were used for the composition of the color image. The dimensionality of the spectral image data obtained by the SRS microscope were first reduced by the PCA (Principal Component Analysis) and then processed by the ICA algorithm [28]. The left side is tumor area and the intermediate layer is the boundary. The right hand side is healthy tissue. You can readily identify Nucleus, White blood cell, Healthy nucleus and so on. At the first glance, you can discriminate between the healthy and tumor areas even in the monochrome image.

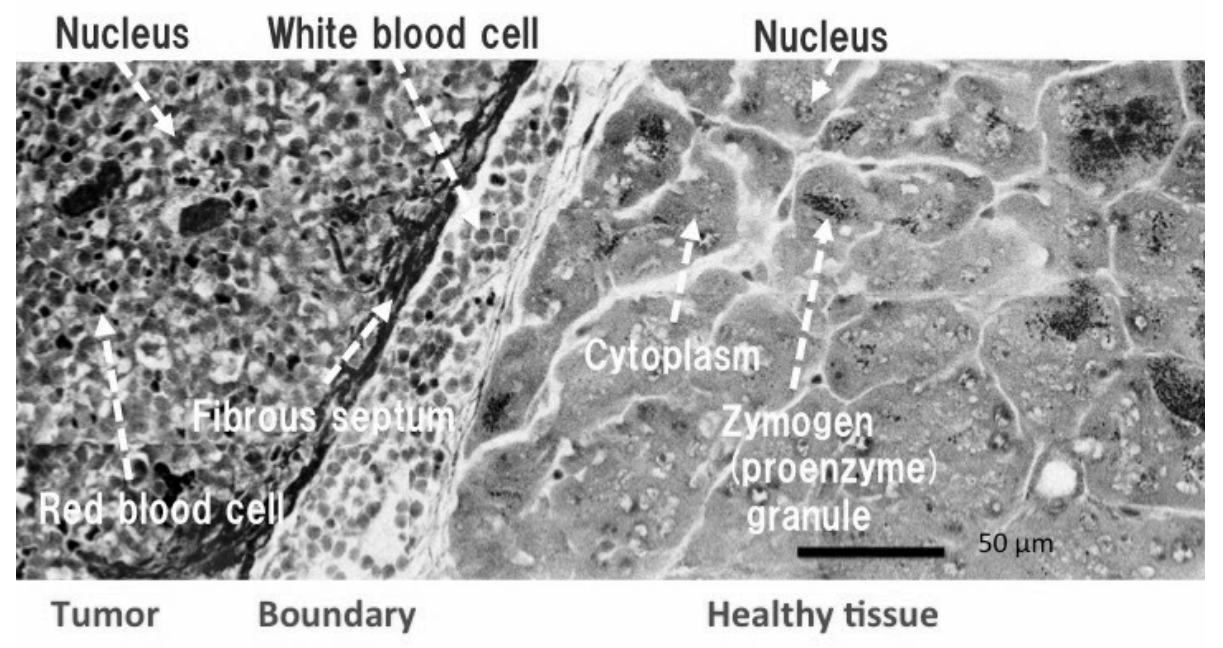

Fig. 7 Color composite ICA image of a mouse pancreas tissue (converted into a monochrome image).

\section{CONCLUSIONS}

The unprecedented progress in computer technology enabled the advanced stage of image processing and manipulation of big and multidimensional data. I have presented high-resolution multi-spectral imaging techniques that can supply us the multidimensional big data for astronomy and medical or life sciences. The former one is now going to be used as the tools for astronomy in space. The latter one is to be used for microscopic analysis of biological tissues. I hope these techniques be the useful eyes of human being for detection of very big and very small substances. It is noted that the optical field concerned with the Fourier transform spectral imaging is located in the far zone of the light source and is locally shift-invariant in the detection area. But the coherence function itself is globally shift-variant and depends on $\boldsymbol{R}$ of $J(\rho)=J(\boldsymbol{R}, \boldsymbol{R}+\boldsymbol{\rho})$ in Eq. (4). Thus, 3D or tomographic imaging is passible by using the Fourier transform spectral imaging technique ingeniously [29].

\section{ACKNOWLEDGEMENT}

A part of this work (Sec 4.3) was conducted in collaboration with Canon Inc. and Prof. Sakamoto of Keio Univ..

\section{REFERENCES}

[1] Michelson, A. A. and Pease, F. G., "Measurement of the diameter of alpha Orionis with the interferometer," Astrophysical Journal, 53, 249-259 (1921). 
[2] Obara, M., Yoshimori, Y. and Itoh, K., "Coherence-based 3D and spectral imaging," submitted to a special issue on "Optics and Photonics for Information Processing," the Asian Journal of Physics (2015).

[3] Freudiger, C. W. et al., "Label-free biomedical imaging with high sensitivity by stimulated Raman scattering microscopy," Science 322, 1857-1861 (2008).

[4] Ozeki, Y., Dake, F., Kajiyama, S., Fukui, K. \& Itoh, K., "Analysis and experimental assessment of the sensitivity of stimulated Raman scattering microscopy," Opt. Express 17, 3651-3658 (2009).

[5] Nandakumar, P., Kovalev, A. \& Volkmer, A., "Vibrational imaging based on stimulated Raman scattering microscopy," New J. Phys. 11, 033026 (2009).

[6] Born, M. and Wolf, E., Principles of Optics, 4th ed., (Pergamon, London) 1970, Chap. 10. or Mandel, L. and Wolf, E., Optical coherence and quantum optics, (Cambridge University Press, Cambridge) 1995, Sec. 4.4.4.

[7] Clark, B. G., "The NRAO tape-recorder interferometer system," Proc. IEEE 61, 1242 (1973).

[8] Chikada, Y. et al., "A $6 \times 320-\mathrm{MHz}$ 1024-channel FFT cross-spectrum analyzer for radio astronomy," Proc. IEEE 75, 1203 (1987).

[9] Wolf, E. and Carter, W. H., "Coherence and radiant intensity in scalar wave fields generated by fluctuating primary planar sources," J. Opt. Soc. Am. 68, 953-964 (1978).

[10] Itoh, K. and Ohtsuka, Y., " Fourier-transform spectral imaging: retrieval of source information from threedimensional spatial coherence," J. Opt. Soc. Am. A 3 (1986), 94.

[11] Mariotti, M. and Ridgway, S. T., "Double Fourier spatio-spectral interferometry-Combining high spectral and high spatial resolution in the near infrared," Astron. Astrophys. 195, 350 (1988).

[12] Shaklan, S. B. and Roddier, F., "Single-mode fiber optics in a long-baseline interferometer," Appl. Opt. 26, 2159 (1987).

[13] Mariotti, J. M., du Foresto, V.C., Perrin, G., Zhao, P., and Léna, P., "Interferometric connection of large ground-based telescopes," Astron. Astrophys. Suppl. Ser. 116, 381 (1996).

[14] Ohta, I. S., Hattori, M. and Matsuo, H., "Development of a multi-Fourier-transform interferometer: fundamentals," Appl. Opt. 45, 2576 (2006).

[15] Ohta, I. S., Hattori, M. and Matsuo, H., "Development of a multi-Fourier-transform interferometer: imaging experiments in millimeter and submillimeter wave bands," Appl. Opt. 46, 2881 (2007).

[16] Itoh, K., in Progress in Optics XXXV, ed. E. Wolf, North-Holland, Amsterdam, 145 (1996).

[17] Leisawitz, D., et al., "The space infrared interferometric telescope (SPIRIT): High-resolution imaging and spectroscopy in the far-infrared," Advances in Space Research 40, 689 (2007).

[18] Rinehart, S.A., et al., "Recent progress in wide-field imaging interferometry," Proc. SPIE 7734, 77342D-1 (2010).

[19] Rizzo, M.J., et al.," Far-Infrared double-Fourier interferometers and their spectral sensitivity,” arXiv: 1507.03961 (2015).

[20] Rizzo, M.J., et al.," Tracking near-infrared fringes on BETTII: a balloon-borne, 8m-baseline interferometer," Proc. SPIE 8445, 84451T-1 (2012).

[21] Leisawitz, D., et al.," Developing wide-field spatio-spectral interferometry for far-infrared space applications," Proc. SPIE 8445, 84450A-1 (2012).

[22] Arimoto, H., Yoshimori, K. and Itoh, K., "Retrieval of the cross-spectral density propagating in free space," J. Opt. Soc. Am. 16, 2447 (1999).

[23] Teeranutranont, S., and Yoshimori, K.," Digital holographic three-dimensional imaging spectrometry,” Appl. Opt. 52, A388 (2013).

[24] Naik, D.N., Pedrini, G., Takeda, M., and Osten, W., "Spectrally resolved incoherent holography: 3D spatial and spectral imaging using a Mach-Zehnder radial-shearing interferometer," Opt. Lett. 39 (2014) 1857.

[25] Ozeki, Y. et al, "High-speed molecular spectral imaging of tissue with stimulated Raman scattering," Nature Photonics 6, 845 (2012).

[26] Ozeki, Y. et al, "Stimulated Raman hyperspectral imaging based on spectral filtering of broadband fiber laser pulses," Opt. Lett. 37, 431 (2012).

[27] Otsuka, Y. et al., presented in The 102nd Annual Meeting of the Japanese Society of Pathology (Collaboration with Canon \& Keio Univ., Prof. Sakamoto).

[28] Satoh, S. et al, "Label-free visualization of acetaminophen-induced liver injury by high-speed stimulated Raman scattering spectral microscopy and multivariate image analysis," Pathology International 64, 518 (2014).

[29] Marks, D.L., et al," Visible Cone-Beam Tomography With a Lensless Interferometric Camera," Science 25, 2164-2166 (1999). 